\title{
In-Silico Screening of Lipid-Based Drug Delivery Systems
}

\author{
Joscha Brinkmann ' (D) - Lara Exner ' • Christian Luebbert ' (1) • Gabriele Sadowski ' (D)
}

Received: 22 July 2020 / Accepted: 9 October 2020 / Published online: 23 November 2020

(C) The Author(s) 2020

\section{ABSTRACT}

Purpose This work proposes an in-silico screening method for identifying promising formulation candidates in complex lipidbased drug delivery systems (LBDDS).

Method The approach is based on a minimum amount of experimental data for API solubilites in single excipients. Intermolecular interactions between APIs and excipients as well as between different excipients were accounted for by the Perturbed-Chain Statistical Associating Fluid Theory. The approach was applied to the in-silico screening of lipid-based formulations for ten model APIs (fenofibrate, ibuprofen, praziquantel, carbamazepine, cinnarizine, felodipine, naproxen, indomethacin, griseofulvin and glibenclamide) in mixtures of up to three out of nine excipients (tricaprylin, Capmul MCM, caprylic acid, Capryol $^{\mathrm{TM}}$ 90, Lauroglycol ${ }^{\mathrm{TM}}$ FCC, Kolliphor TPGS, polyethylene glycol, carbitol and ethanol).

Results For eight out of the ten investigated model APIs, the solubilities in the final formulations could be enhanced by up to 100 times compared to the solubility in pure tricaprylin. Fenofibrate, ibuprofen, praziquantel, carbamazepine are recommended as type I formulations, whereas cinnarizine and felodipine showed a distinctive solubility gain in type II formulations. Increased solubility was found for naproxen and indomethacin in type IIIb and type IV formulations. The solubility of griseofulvin and glibenclamide could be slightly enhanced in type IIIb formulations. The experimental validation agreed very well with the screening results.

Conclusion The API solubility individually depends on the choice of excipients. The proposed in-silico-screening approach

Gabriele Sadowski

gabriele.sadowski@tu-dortmund.de

TU Dortmund University, Laboratory of Thermodynamics, Emil-Figge-Str. 70, D-44227 Dortmund, Germany allows formulators to quickly determine most-appropriate types of lipid-based formulations for a given API with low experimental effort.

KEY WORDS lipid-based formulations · PC-SAFT . solubility · thermodynamic modeling

\section{NOMENCLATURE}

a Helmholtz energy

h molar enthalpy

$c_{p}$ heat capacity

M molar mass

m segment number

$k_{B} \quad$ Boltzmann constant

$k_{i i} \quad$ binary interaction parameter

$\mathrm{N}$ number of data points

$N_{\text {assoc }}$ number of association sites

$R \quad$ ideal gas constant

P pressure

$\mathrm{T}$ temperature

u dispersion energy

$\mathrm{w}_{\mathrm{i}} \quad$ mass fraction

$x_{i} \quad$ mole fraction

$$
\begin{aligned}
& \mathrm{J} \mathrm{mol}^{-1} \\
& \mathrm{~mJ} \mathrm{~mol}^{-1} \\
& \mathrm{~J}\left(\mathrm{~mol} \mathrm{~K}^{-1}\right. \\
& \mathrm{g} \mathrm{mol}^{-1} \\
& - \\
& \mathrm{J} \mathrm{K}^{-1} \\
& - \\
& - \\
& - \\
& \mathrm{J}(\mathrm{mol} \mathrm{K})^{-1} \\
& \text { bar } \\
& \mathrm{K},{ }^{\circ} \mathrm{C} \\
& \mathrm{J}
\end{aligned}
$$

\section{GREEK CHARACTERS}

Y activity coefficient -

$\varepsilon^{A B B i}$ association energy $J$.

$\rho \quad$ density $\mathrm{kg} \mathrm{m}^{-3}$

$\mathrm{K}^{\mathrm{AiBi}}$ association volume -

$\sigma_{\text {seg }}$ segment diameter $\AA$

\section{SUBSCRIPTS}

i,j component

int intersection

\section{SUPERSCRIPTS}




$\begin{array}{ll}\text { assoc } & \text { associating } \\ \text { disp } & \text { dispersion } \\ \text { hc } & \text { hard chain } \\ \text { L } & \text { liquid } \\ \text { res } & \text { residual } \\ \text { S } & \text { solid } \\ \text { V } & \text { vapor }\end{array}$

\section{INTRODUCTION}

Many of the newly-developed active pharmaceutical ingredients (APIs) possess an insufficient solubility in water. If they cannot be administered as conventional tablets providing sufficient bioavailability, other formulation strategies must be explored to overcome this limitation. Promising alternatives in this field are lipid-based drug delivery systems (LBDDS), in which the APIs are dissolved in liquid formulations. Since the API is administered in a dissolved state, LBDDS can avoid the slow API-dissolution step in the human body and increase the API bioavailability. [1-4] In their simplest form, LBDDS comprise the API dissolved in a pure triglyceride (TG). More often, LBDDS contain complex mixtures of excipients such as glycerides, surfactants or cosolvents to further enhance the API solubility. [5-7].

The API solubility in the liquid excipient (mixture) is a key property for the development of LBDDS. [8] Therefore recent works focused on API-solubility measurements in either TGs [9-11], natural edible oils [11-13], or other commercial excipients $[14,15]$. The most-common experimental methods to measure API solubilities are differential scanning calorimetry (DSC) [9, 13, 16], Raman spectroscopy [9, 13], UV-vis [14, 17], and high-performance liquid chromatography (HPLC) [9, 13, 15].

Guidelines to develop LBDDS were discussed in recent works e.g. by Holm [8], Kuentz [5] and Williams et al. [18]. According to these works, the first step in the development of an LBDDS is to find out whether or not an API is highly soluble in excipients or mixtures thereof. The large number of commercially available excipients yields numerous combinatory possibilities to formulate an API in a LBDDS.

Pouton [6, 7] developed a lipid formulation classification system (LFCS), which considers different aspects such as aqueous dispersion, digestion and absorption behavior, and suggested promising formulation compositions for further in vitro and in vivo tests. [7, 19] Although the LFCS helps formulators to roughly orientate in the wide field of LBDDS formulations, finding an appropriate excipient mixture for LBDDS still requires countless trial-and-error experiments and is therefore very time consuming and expensive. Thus, there is a strong demand for predictive in-silico methods, which can drastically reduce the experimental effort.
Along this line, this work proposes applying a thermodynamic model, namely the Perturbed-Chain Statistical Associating Fluid Theory (PC-SAFT), for predictive LBDDS-screening purposes [20]. PC-SAFT has already been successfully applied to model API solubilities in pure TGs [9], in natural edible oils [13], and in other excipients [17, 21]. Based on model parameters for pure APIs and excipients as well as for binary mixtures thereof, an in-silico screening is applied in this work to identify excipient mixtures consisting of up to three different excipients providing the highest API solubility. The in-silico screening is then validated via measuring the API solubilities in the proposed formulations.

\section{DEFINITION OF LBDDS FORMULATION WINDOWS USING THE LIPID FORMULATION CLASSIFICATION SYSTEM}

The LFCS developed by Pouton [6, 7] is as an empirical framework defining reasonable excipient compositions of a LBDDS. It was considered in this work to ensure that the insilico solubility screening only comprises reasonable excipient compositions. Table I summarizes the four possible formulation classes according to the LFCS and the utilizable excipient compositions in each formulation class. In this work the term excipient mixture describes a mixture of excipients without the API, whereas formulation stands for a mixture of API and excipients.

The LFCS categorizes LBDDS into four formulation types, while formulation type III is subdivided into two subclasses. The simplest formulations (type I) comprise only an API dissolved in a TG (e.g. TG8 $\left.8_{0} 8_{0} 8_{0}\right)$ or in a mixture of MGs and DGs (Capmul MCM). If the API solubility in type I excipients is found to be too low, 20-60 wt.\% hydrophobic surfactants (Capryol 90, Lauroglycol FCC, MC8 ${ }_{0}$ ) might be added (type II formulation). More hydrophilic APIs not sufficiently dissolvable in type I or type II formulations are candidates for type IIIa and IIIb formulations. These formulations become more hydrophilic and thus comprise high contents of hydrophilic surfactants (TPGS1000) and cosolvents (carbitol, ethanol, polyethylene glycol 400 (PEG400)). Type IV formulations finally do not contain any glycerides anymore but only APIs, surfactants and cosolvents. $[6,7]$.

\section{MATERIALS AND METHODS}

\section{Materials}

The APIs fenofibrate (FFB), ibuprofen (IBU), indomethacin (IND) and felodipine (FEL) with purity $>98 \%$ were purchased from TCI (Tokyo, Japan). Cinnarizine (CIN), griseofulvin (GRI), glibenclamide (GLI) were purchased from Alfa Aesar 
Composition of the excipient mixture (wt\%)

\begin{tabular}{|c|c|c|c|c|c|}
\hline & \\
\hline & Type I & Type II & Type Illa & Type IIIb & Type IV \\
\hline TGs or mixed MGs and DGs: $T G 8_{0} 8_{0} 8_{0}$, Capmul MCM & 100 & $40-80$ & $40-80$ & $<20$ & 0 \\
\hline Hydrophobic surfactants (HLB < I 2): Capryol 90, Lauroglycol FCC, MC8 0 & 0 & $20-60$ & 0 & 0 & $0-20$ \\
\hline Hydrophilic surfactants (HLB > I 2): TPGSI 000 & 0 & 0 & $20-40$ & $20-50$ & $30-80$ \\
\hline $\begin{array}{l}\text { Hydrophilic cosolvents: } \\
\text { Carbitol, ethanol, PEG400 }\end{array}$ & 0 & 0 & $0-40$ & $20-50$ & $0-50$ \\
\hline
\end{tabular}

(Karlsruhe, Germany) possessing a purity $>97 \%$. Tricaprylin $\left(\mathrm{TG}_{0} \mathrm{8}_{0} \mathrm{8}_{0}\right)$ and carbitol were purchased from Sigma Aldrich (Steinheim, Germany) with purity $>99 \%$. The glycerides are abbreviated in this work according to [22] (e.g. tricaprylin: $\mathrm{TG}_{0} 8_{0} 8_{0}$, monolaurin: $\mathrm{MG}_{0}$ : The first two letters define the type of component $(\mathrm{TG}=$ triglyceride, $\mathrm{DG}=$ diglyceride and $\mathrm{MG}=$ monoglyceride. The capital numbers give the number of carbon atoms in each carbon chain and the subscript denotes the number of unsaturated bonds in each carbon chain.

Caprylic acid $\left(\mathrm{MC}_{0}\right)$ was obtained from Merck (Darmstadt, Germany) with purity 99\%. Capryol 90 TM (Capryol 90) was provided by Gattefosse (Saint-Priest Cedex, France). Kolliphor® TPGS (TPGS1000) was provided by BASF (Ludwigshafen, Germany). The surfactants Capryol 90 and TPGS1000 were taken from the same batches as in a recent work [17]. Water was purified by Milli-Q from Merck Millipore (Darmstadt, Germany). Acetonitrile used for the mobile phase in the HPLC was of analytical grade with minimal purity of $99.9 \%$ from VWR Chemicals (Darmstadt, Germany). Phosphoric acid was purchased from Sigma Aldrich with purity $>99.9 \%$.

\section{Experimental Methods}

The API solubility in an excipient or excipient mixture at given temperature (temperature accuracy $\pm 0.1 \mathrm{~K}$ ) was determined by equilibrating an excess amount of API in the respective excipient or excipient mixture for at least three days. The saturated liquid phase was then filtered to remove API crystals and diluted with acetonitrile to prevent API crystallization during further handling. The API content in the saturated liquid phase was analyzed with HPLC. An Agilent 1200 HPLC (Santa Clara, USA) with a ZOBRAX Eclipse XDBC18 reversed-phase column from Agilent (Santa, Clara) was used for this purpose. The column temperature was $35{ }^{\circ} \mathrm{C}$. An acetonitrile/water mixture (70/ $30 \mathrm{v} / \mathrm{v})$ with phosphoric acid $(\mathrm{pH}=2.5)$ was utilized as the mobile phase. The mobile phase had a flow rate of
$1 \mathrm{~mL} \min ^{-1}$. All APIs were quantified by a UV-vis detector. FFB, and GLI were analyzed at a wavelength of $286 \mathrm{~nm}$; IBU, FEL, IND, PZQ, CIN, and GRI were analyzed at a wavelength of $225 \mathrm{~nm}$. The excipients were checked not to overlap the UV-vis signal of the APIs at these wavelengths. All samples were measured in triplicates in the HPLC.

The API solubilities were determined in two independent experiments with an averaged uncertainty of $0.64 \%$.

\section{Solubility Modeling with PC-SAFT}

The solubility of a solute $\mathrm{i}$ (for instance an API) in glycerides or other excipients is calculated using Eq. 1. [23]

$x_{i}=\frac{1}{\gamma_{i}} \cdot \exp \left[-\frac{\Delta h_{i}^{S L}}{R \cdot T} \cdot\left(1-\frac{T}{T_{i}^{S L}}\right)-\frac{\Delta c_{p i}^{S L}}{R}\left[\ln \left(\frac{T_{i}^{S L}}{T}\right)-\frac{T_{i}^{S L}}{T}+1\right]\right]$

Here, $x_{A P I}$ is the mole-fraction solubility of the API in the liquid phase. The API activity coefficient $\gamma_{A P I}$ considers all intermolecular interactions among the solute $\mathrm{i}$ and glycerides/excipients and was calculated in this work using PC-SAFT. The melting properties of the solute are the melting temperature $\left(T_{A P I}^{S L}\right)$, the melting enthalpy $\left(\Delta h_{A P I}^{S L}\right)$, and the difference of the heat capacities of the solid and liquid API $\left(\Delta c_{p, A P I}^{S L}\right)$. Table II contains the melting properties of all APIs considered in this work.

PC-SAFT approximates a molecule as a chain of $\mathrm{m}_{\text {seg }}$ spherical segments possessing a defined diameter $\left(\sigma_{\text {seg }}\right)$. Dispersive van-der-Waals forces between segments of different molecules are accounted by the dispersiveenergy parameter $\mathrm{u}_{\mathrm{i}} \mathrm{k}_{\mathrm{B}}^{-1}\left(\mathrm{k}_{\mathrm{B}}\right.$ is the Boltzmann constant). The association energy parameter $\varepsilon_{\mathrm{i}}{ }^{\mathrm{AiBi}} \mathrm{k}_{\mathrm{B}}{ }^{-1}$ and the association volume $\kappa^{\mathrm{AiBi}}$ consider associative forces (e.g. hydrogen bonding) between molecules. Thus, up to five parameters are used to describe the behavior of a pure component, e.g. API or excipient [30]. The pure component parameters used in this work were already available and are summarized in Tables III and IV. 
Table II Melting temperature, melting enthalpy and heat capacity difference between liquid and crystalline state of APIs and TPGSI000

\begin{tabular}{|c|c|c|c|c|}
\hline Component & $T_{i}^{S L}[\mathrm{~K}]$ & $\Delta h_{i}^{S L}\left[\mathrm{KJ} \mathrm{mol}{ }^{-1}\right]$ & $\Delta c_{p, i}^{S L}\left[\mathrm{~J} \mathrm{~mol}^{-1} \mathrm{~K}^{-1}\right]$ & Source \\
\hline FFB & 354.0 & 33.5 & 124.3 & {$[9,24]$} \\
\hline $\mathrm{IBU}$ & 350.2 & 25.5 & 50.3 & {$[25]$} \\
\hline IND & 433.3 & 39.3 & 117.0 & {$[2 \mid]$} \\
\hline CBZ & 448.0 & 26.8 & 65.2 & {$[26]$} \\
\hline GRI & 491.2 & 32.8 & 93.8 & {$[27]$} \\
\hline GLI & 446.5 & 52.9 & 153.6 & {$[28]$} \\
\hline NAP & 429.5 & 31.5 & 87.4 & {$[28]$} \\
\hline $\mathrm{CIN}$ & 394.0 & 37.1 & 113.6 & [29] \\
\hline PZQ & 411.5 & 28.4 & 103.3 & [Unpublished] \\
\hline FEL & 416.9 & 30.8 & 89.9 & {$[25]$} \\
\hline TPGSI000 & 311.7 & | 56.2 & - & [Unpublished] \\
\hline
\end{tabular}

The segment diameter and the dispersion energy in mixtures are calculated using the Berthelot-Lorentz combining rules (Eqs. 2 and 3).

$\sigma_{i j}=\frac{1}{2}\left(\sigma_{i}+\sigma_{j}\right)$

$u_{i j}=\left(I-k_{i j}\right) \cdot \sqrt{u_{i} \cdot u_{j}}$

In Eq. 3, a binary interaction parameter $\left(k_{i j}\right)$ is introduced when calculating the dispersion energy. It corrects for deviations from the postulated mixing rules for interactions between unlike segments [20]. The $k_{i j}$ value may linearly depend on temperature (Eq. 4).

$k_{i j}(T)=k_{i j, s l o p e} \cdot T[K]+k_{i j, i n t}$

Binary interaction parameters $k_{i j}$ used in this work were taken from literature and are summarized in Tables V and VI.

\section{SCREENING RESULTS}

All screening results obtained in this work for API solubilities in formulations are full predictions based on the PC-SAFT parameters obtained for pure components and binary systems (see previous section). The screening was performed by comparing the PC-SAFT-predicted API solubilities in excipient mixtures of different formulation types (Table I). A more-complex formulation type was only recommended, when leading to a remarkable increase in API solubility compared to a less-complex one.

\section{Type I Formulations}

Type I formulations are the simplest LFCS formulations. They comprise mixtures of APIs and glycerides. Figure 1 shows the PC-SAFT calculated solubilities of the APIs investigated in this work in $\mathrm{TG}_{0} 8_{0} \mathrm{8}_{0}$ and Capmul MCM at $25^{\circ} \mathrm{C}$. The API solubilities in $\mathrm{TG}_{0} 8_{0} 8_{0}$ were calculated using the binary parameters from Table V. Capmul MCM is a mixture
Table III PC-SAFT purecomponent parameters of the APIs investigated in this work

\begin{tabular}{|c|c|c|c|c|c|c|c|c|}
\hline API & $\begin{array}{l}\mathrm{M} \\
{\left[\mathrm{g} \mathrm{mol}^{-1}\right]}\end{array}$ & $\begin{array}{l}\mathrm{m}_{\mathrm{seg}} \\
{[-]}\end{array}$ & $\begin{array}{c}\sigma_{\mathrm{seg}} \\
{[\AA]}\end{array}$ & $\begin{array}{l}\mathrm{u}_{\mathrm{i}} \mathrm{k}_{\mathrm{B}}{ }^{-1} \\
{[\mathrm{~K}]}\end{array}$ & $\begin{array}{l}\varepsilon_{\mathrm{i}}^{\mathrm{AiBi}} k_{\mathrm{B}}^{-1} \\
{[\mathrm{~K}]}\end{array}$ & $\begin{array}{l}\mathrm{K}^{\mathrm{AiBi}} \\
{[-]}\end{array}$ & $\begin{array}{l}N_{\text {assoc }} \\
{[-]}\end{array}$ & Source \\
\hline FFB & 360.80 & 3.859 & 4.767 & 244.8 & 0.0 & 0.02 & $0 / 2$ & [9] \\
\hline $\mathrm{IBU}$ & 206.28 & 2.522 & 4.432 & 374.7 & 879.4 & 0.03 & $2 / 2$ & {$[3 \mid]$} \\
\hline IND & 357.79 & 14.283 & 3.535 & 262.8 & 886.4 & 0.02 & $3 / 3$ & {$[2 \mid]$} \\
\hline CBZ & 236.27 & 9.978 & 2.658 & 151.6 & I094.1 & 0.02 & $|/|$ & [26] \\
\hline GRI & 352.77 & 14.174 & 3.372 & 221.3 & 1985.5 & 0.02 & $2 / 2$ & {$[27]$} \\
\hline GLI & 494.00 & | 8.278 & 3.058 & 221.1 & 2181.9 & 0.02 & $3 / 3$ & [28] \\
\hline NAP & 230.26 & 8.110 & 2.939 & 229.5 & 934.2 & 0.02 & $2 / 2$ & [28] \\
\hline $\mathrm{CIN}$ & 368.51 & $|3.56|$ & 3.086 & 231.0 & 983.4 & 0.02 & $1 / \mid$ & [29] \\
\hline PZQ & 312.41 & 6.221 & 4.090 & 327.1 & 0.0 & 0.02 & $0 / 2$ & [Unpublished] \\
\hline FEL & 384.26 & | 1.528 & 3.205 & 234.5 & | 58|.| & 0.02 & $2 / 2$ & {$[25]$} \\
\hline
\end{tabular}


Table IV PC-SAFT purecomponent parameters of excipients investigated in this work

\begin{tabular}{|c|c|c|c|c|c|c|c|c|}
\hline & $\begin{array}{l}\mathrm{M} \\
{\left[\mathrm{g} \mathrm{mol}^{-1}\right]}\end{array}$ & $\begin{array}{l}m_{\mathrm{seg}} \\
{[-]}\end{array}$ & $\begin{array}{l}\sigma_{\text {seg }} \\
{[\AA]}\end{array}$ & $\begin{array}{l}\mathrm{u}_{\mathrm{i}} \mathrm{k}_{\mathrm{B}}{ }^{-1} \\
{[\mathrm{~K}]}\end{array}$ & $\begin{array}{l}\varepsilon_{i}^{A i B i} k_{B}^{-1} \\
{[K]}\end{array}$ & $\begin{array}{l}\mathrm{K}^{\mathrm{AiBi}} \\
{[-]}\end{array}$ & $\begin{array}{l}N_{\text {assoc }} \\
{[-]}\end{array}$ & Source \\
\hline TG $8_{0} 8_{0} 8_{0}$ & 470.69 & 9.482 & 4.235 & 281.6 & 0 & 0.020 & $3 / 3$ & {$[22]$} \\
\hline $\mathrm{DG} 1 \mathrm{O}_{0} 10_{0}$ & 400.60 & 10.965 & 3.767 & 242.1 & 4167.5 & 0.010 & $1 / 1$ & [Unpublished] \\
\hline$M G 12_{0}$ & 274.40 & 7.931 & 3.738 & 235.8 & 3475.4 & 0.010 & $2 / 2$ & [Unpublished] \\
\hline MG8o & 218.29 & 5.107 & 3.809 & 236.2 & 3475.4 & 0.010 & $2 / 2$ & [Unpublished] \\
\hline$M C 8_{0}$ & 144.21 & 5.306 & 3.505 & 255.9 & 2635.3 & 0.015 & $1 / 1$ & [Unpublished] \\
\hline Lauroglycol FCC & 258.40 & 6.848 & 3.932 & 290.3 & 2092.8 & 0.020 & $1 / 1$ & {$[17]$} \\
\hline Capryol 90 & 220.31 & 6.369 & 3.714 & 275.8 & 1405.8 & 0.020 & $\mathrm{I} / \mathrm{I}$ & {$[17]$} \\
\hline TPGSI000 & 1513.00 & 50.988 & 3.421 & 254.4 & 3073.0 & 0.020 & $1 / 1$ & {$[17]$} \\
\hline carbitol & 134.18 & 4.883 & 3.387 & 239.2 & 2360.5 & 0.019 & $1 / 1$ & [Unpublished] \\
\hline PEG400 & 400.00 & 20.240 & 2.899 & 204.6 & | 799.8 & 0.020 & $1 / 1$ & {$[32]$} \\
\hline ethanol & 46.07 & 2.3827 & 3.177 & 198.2 & 2653.4 & 0.032 & $1 / 1$ & {$[30]$} \\
\hline
\end{tabular}

of different MGs and DGs (mainly $\mathrm{MG}_{0}$, with lower amounts of $\mathrm{MG} 10_{0}, \mathrm{DG}_{0} 8_{0}$ and $\left.\mathrm{DG} 10_{0} 10_{0}\right)$. Due to the complex composition of Capmul MCM, which might differ from batch to batch, the most-prominent components were used for the modeling [13]. Thus, in this work, Capmul MCM was assumed to contain $90 \mathrm{wt}^{\%}$ monocaprylin $\left(\mathrm{MG}_{0}\right)$ and $10 \mathrm{wt}^{\%} \%$ dicaprin $\left(\mathrm{DG} 10_{0} 10_{0}\right)$.

The solubilities of FFB $\left(9.5 \mathrm{wt}^{\mathrm{0}} \%\right.$ ) and IBU $\left(10.6 \mathrm{wt}^{\mathrm{\%}} \%\right)$ in $\mathrm{TG} 8_{0} 8_{0} 8_{0}$ were calculated to be high compared to the ones of the other APIs. CIN, FEL and PZQ have solubilities between $1.1 \mathrm{wt} \%$ and $3.2 \mathrm{wt} \%$ whereas the solubility of CBZ, GLI, GRI, IND, and NAP is even below $1 \mathrm{wt} \%$. These low solubilities make the last-mentioned APIs unfavorable to be formulated in pure $\mathrm{TG}_{0} \mathrm{8}_{0} 8_{0}$.

The predicted solubilities of CBZ and PZQ in Capmul MCM are very high compared to those in $\mathrm{TG}_{0} 8_{0} 8_{0}$. They are increased by $6000 \%$ (CBZ) and $1200 \%$ (PZQ) compared to the ones in $\mathrm{TG}_{0} 8_{0} 8_{0}$. Also, the solubilities of FEL and IBU in Capmul MCM were predicted to be remarkably enhanced compared to $\mathrm{TG}_{0} 8_{0} 8_{0}$. In contrast, the FFB solubility in Capmul MCM was predicted to be $6.9 \mathrm{wt} \%$ which is a decrease by $28 \%$ compared to the one in $\mathrm{TG}_{0} 8_{0} 8_{0}$. For all other APIs, the solubilities in Capmul MCM were predicted to slightly increase compared to the ones in $\mathrm{TG}_{0} \mathrm{8}_{0} \mathrm{8}_{0}$, but to not exceed $2.2 \mathrm{wt} \%$.

As a result of this screening step, FFB is recommended to be formulated in $\mathrm{TG}_{0} 8_{0} 8_{0}$. The high solubilities of $\mathrm{CBZ}$, FEL, and PZQ in Capmul MCM suggest that they are promising candidates for type I formulations with Capmul MCM or other MG/DG mixtures. IBU reveals high solubilities in $\mathrm{TG}_{0} 8_{0} \mathrm{8}_{0}$ as well as in Capmul MCM.

\section{Type II Formulations}

The solubility of many APIs in type I formulations is quite low (Fig. 1). Thus, the second screening step was to check, whether the addition of a hydrophobic surfactant (type II formulation) can enhance the API solubility in the TG. Figure 2 compares CIN and FEL solubilities in TG8 $8_{0} 8_{0} 8_{0}+$ hydrophobic surfactant mixtures (surfactants being either $\mathrm{MC}_{0}$, Capryol 90, or Lauroglycol FCG).

The screening results in Fig. 2a show, that the solubility of $\mathrm{CIN}$ in $\mathrm{TG}_{0} 8_{0} 8_{0}$ strongly increases when adding $\mathrm{MC}_{0}$ to $\mathrm{TG}_{0} 8_{0} 8_{0}$ which was experimentally validated for a mixture of $60 \mathrm{wt} \% \mathrm{TG}_{0} 8_{0} 8_{0}$ and $40 \mathrm{wt} \% \mathrm{MC}_{0}$ (CIN solubility of $23.1 \mathrm{wt}^{\%} \%$ ). In contrast, the predictions did not reveal a solubility gain upon addition of Capryol 90 or Lauroglycol FCG to $\mathrm{TG}_{0} 8_{0} 8_{0}$.

The FEL solubility (Fig. 2b) in $\mathrm{TG}_{0} \mathrm{8}_{0} \mathrm{8}_{0}$ was predicted to increase upon addition of Capryol 90. E.g., in an excipient mixture of $60 \mathrm{wt} \% \mathrm{TG}_{0} 8_{0} 8_{0}$ and $40 \mathrm{wt} \%$ Capryol 90, the FEL solubility was predicted to be $6.7 \mathrm{wt} \%$, which very well agrees with the experimental value $(7.5 \mathrm{wt} \%)$. In contrast to that, the screening suggests that addition of $\mathrm{MC}_{0}$ does not enhance the FEL solubility in $\mathrm{TG}_{0} \mathrm{8}_{0} \mathrm{8}_{0}$.

FFB and IBU already revealed high solubilities in $\mathrm{TG}_{0} 8_{0} 8_{0}$ (Fig. 1). Still, it was investigated whether an addition of a hydrophobic surfactant could further enhance the solubilities of these APIs. Figure 3 thus displays the predicted solubilities of FFB and IBU in mixtures of TG $8_{0} 8_{0} 8_{0}$ and one of the hydrophobic surfactants Capryol 90, MC $_{0}$ or Lauroglycol FCG.

The screening results from Fig. 3a clearly show that the FFB solubility is predicted to only slightly increase when adding hydrophobic surfactants to $T G 8_{0} 8_{0} 8_{0}$. For adding Lauroglycol FCC, even a slight decrease in FFB solubility was predicted. The experimental FFB solubility in an excipient mixture of $60 \mathrm{wt} \% \mathrm{TG}_{0} 8_{0} 8_{0}$ and $40 \mathrm{wt} \%$ Capryol $90 \mathrm{was}$ afterwards determined to be $13.2 \mathrm{wt} \%$ and very well agrees with the prediction.

The screening for IBU (Fig. 3b) shows a distinctively higher solubility in a mixture containing $\mathrm{MC}_{0}$ or Capryol 90 compared to the IBU solubility in pure $\mathrm{TG}_{0} 8_{0} 8_{0}$. The predicted IBU solubility for an excipient mixture of $60 \mathrm{wt} \% \mathrm{TG}_{0} 8_{0} 8_{0}$ 


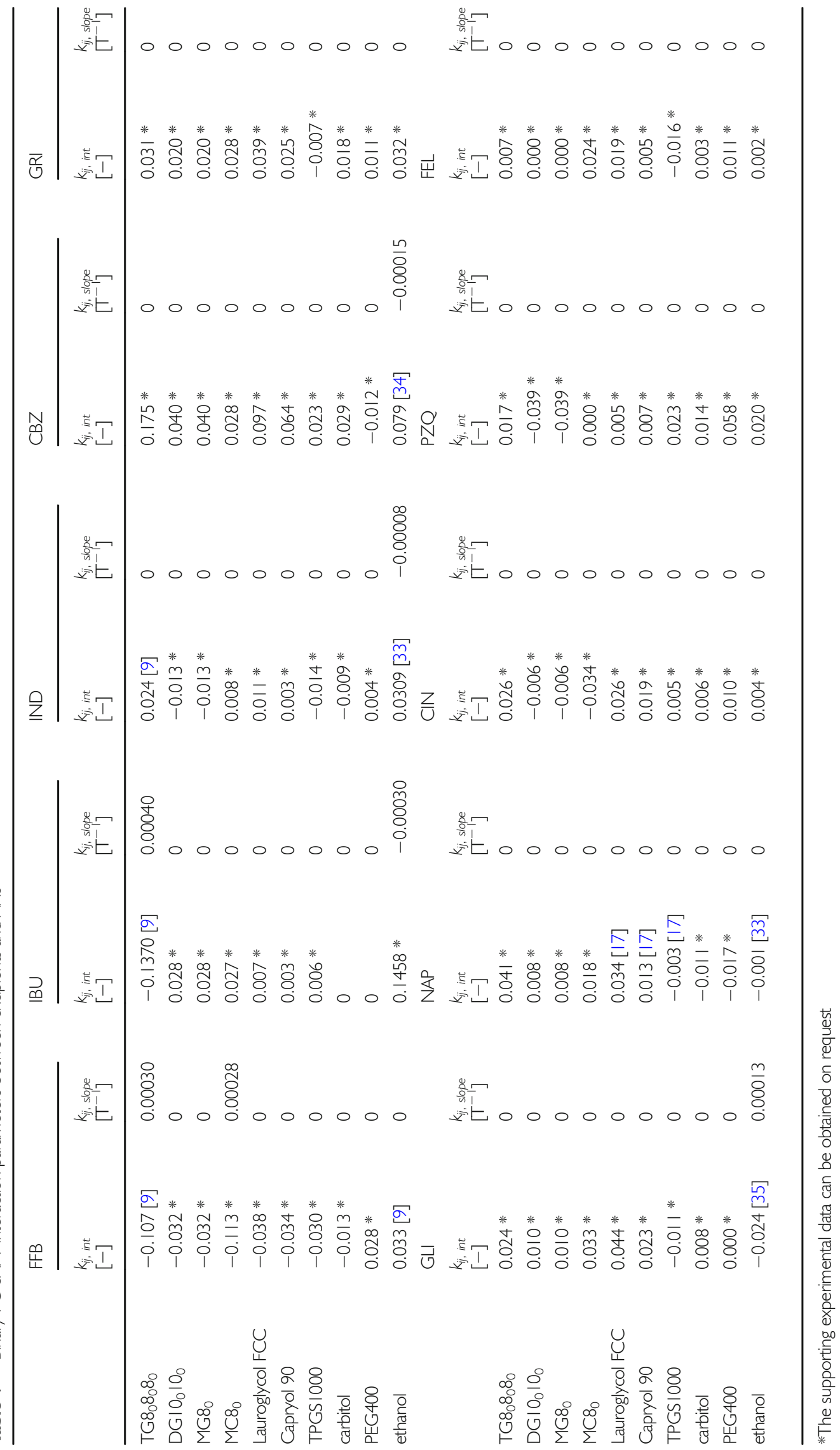


Table VI Binary PC-SAFT interaction parameter $k_{i j}$ in between the excipients TPGSI 000, TG $8_{0} 8_{0} 8_{0}$ and carbitol [Unpublished]

\begin{tabular}{lllll}
\hline Component i/j & TPGSI000 & TG8 $8_{0} 8_{0} 8_{0}$ & carbitol & ethanol \\
\hline TPGSI000 & 0.0120 & -0.0100 & 0.0000 \\
TG $8_{0} 8_{0} 8_{0}$ & & 0.0235 & 0.0300 \\
\hline
\end{tabular}

and $40 \mathrm{wt} \% \mathrm{MC}_{0}$ is $24.3 \mathrm{wt}^{\circ} \%$. The experimental validation revealed an IBU solubility of $21.3 \mathrm{wt} \%$, which again very well agrees with the screening result.

The PZQ solubilities in $\mathrm{TG}_{0} \mathrm{8}_{0} \mathrm{8}_{0}$ and in Capmul MCM differ remarkably (see Fig. 1). Figure 4 now compares the screening results for the PZQ solubility in type II formulations based on (a) $\mathrm{TG}_{0} 8_{0} 8_{0}$ and (b) Capmul MGM.

The low PZQ solubility in $T G 8_{0} 8_{0} 8_{0}$ was predicted to increase upon addition of any of the investigated hydrophobic surfactants (Fig. 4a). The highest solubility was predicted for excipient mixtures containing $\mathrm{TG}_{0} \mathrm{8}_{0} \mathrm{8}_{0}$ and $\mathrm{MC}_{0}$ whereas it was found to be lowest in excipient mixtures of $\mathrm{TG}_{0} 8_{0} 8_{0}$ and Lauroglycol FCC. Noticeably, all solubilities screened in Fig. 4a are lower than the PZQ solubility in pure Capmul MCM (Figs. 1 and 4b). Moreover, it can be seen that addition of hydrophobic surfactants to Capmul MCM was predicted to even decrease the PZQ solubility (Fig. 4b).

For all investigated APIs, the solubility was found to be lower in type II formulations containing Lauroglycol FCC compared to those containing Capryol 90. It can thus be concluded that Capryol 90 is the preferred excipient in mixtures with $\mathrm{TG}_{0} 8_{0} \mathrm{8}_{0}$ and Capmul MCM as already postulated in an earlier work [17].

Moreover, the screening results revealed that CIN should be formulated in a mixture of $\mathrm{TG}_{0} 8_{0} 8_{0}$ and $\mathrm{MC} 8_{0}$. FEL solubility is highest in an excipient mixture of TG $8_{0} 8_{0} 8_{0}$ and

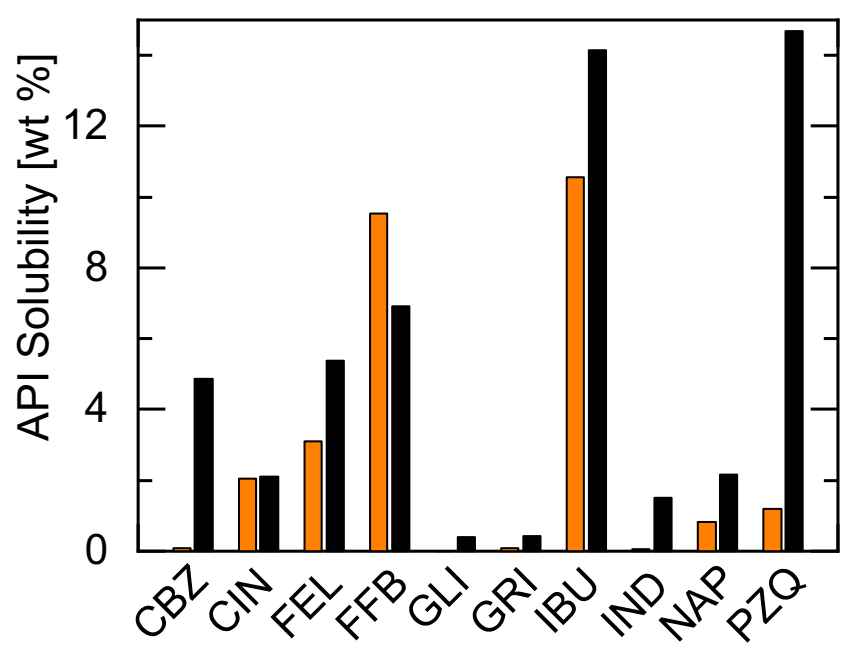

Fig. I PC-SAFT API solubilities in type I formulations at $25^{\circ} \mathrm{C}$. The left bars are API solubilities in $\mathrm{TG} 8_{0} 8_{0} 8_{0}$, the right bars are $\mathrm{API}$ solubilities in Capmul MCM.
Capryol 90. Thus, both APIs are promising candidates for type II formulations. FFB and IBU are sufficiently soluble in every single excipient, even in pure $\mathrm{TG}_{0} 8_{0} \mathrm{8}_{0}$ (see Fig. 1) and therefore should be preferably formulated as type I formulations. Only if the solubility of IBU in $\mathrm{TG}_{0} 8_{0} 8_{0}$ needs to be further increased, this could be achieved by adding $\mathrm{MC}_{0}$ or Capryol 90. PZQ is suggested to be formulated in mixtures of MGs, DGs and carboxylic acids.

\section{Type III Formulations}

Some APIs show low solubilities in type I and type II formulations. For instance, the screening revealed that the IND solubility does not exceed 2 wt.\% in formulations of type I or II. Thus, it was reasonable to proceed the screening for this API and to predict IND solubilities in type IIIa and IIIb formulations. According to the LFCS, these formulation types contain high amounts of hydrophilic surfactant and cosolvent and lower amounts of glycerides (see Table I). The LFCS formulation window shown in the diagram visualizes reasonable compositions for the in-silico screening.

Figure 5 shows the predicted IND solubilities in a mixture of TG8 $8_{0} 8_{0} 8_{0}$, TPGS 1000 (hydrophilic surfactant), and carbitol (cosolvent) at $25{ }^{\circ} \mathrm{C}$. The solubility in pure $\mathrm{TG}_{0} \mathrm{8}_{0} \mathrm{8}_{0}$ is quite low $\left(0.1 \mathrm{wt}^{\%} \%\right)$ and increases to more than $10 \mathrm{wt} \%$ for higher amounts of carbitol. Besides the API, TPGS1000 can crystallize at $25{ }^{\circ} \mathrm{C}$. This was considered here and is also visualized in Fig. 5. The region where TPGS1000 is predicted to crystallize in the excipient mixture intersects with the concentration window of type IIIa formulations. In this area, the excipient mixture is not completely liquid and should therefore not be considered for an LBDDS.

The screening results suggest that the IND solubility in type IIIa formulations is considerably lower than in Type IIIb formulations which moreover will not suffer from TPGS1000 crystallization throughout the entire formulation window. Thus, for IND, type IIIb formulations are more promising than IIIa formulations.

\section{Type IV Formulations}

Type IV formulations are considered for those APIs where the addition of glycerides like $\mathrm{TG}_{0} 8_{0} \mathrm{8}_{0}$ to the excipient mixture leads to a decrease in API solubility. Formulations of this type do not contain any lipid, but a mixture of hydrophilic and hydrophobic surfactants and cosolvents (Table I). According to Fig. 5, the maximum IND solubility was predicted to be $9.3 \mathrm{wt}^{\%} \%$ in a TG-free mixture of $50 \mathrm{wt} \%$ TPGS1000 and $50 \mathrm{wt} \%$ carbitol (in perfect agreement with the experimental validation of $10.2 \mathrm{wt} \%$ ). This is a solubility gain of more than $9000 \%$ compared to the IND solubility in pure $\mathrm{TG}_{0} 8_{0} 8_{0}$. For this example, it was checked whether the addition of 
Fig. 2 Solubility of (a) $\mathrm{CIN}$ and (b) FEL in mixtures of $\mathrm{TG} 8_{0} 8_{0} 8_{0}$ and either MC80, Capryol 90, or Lauroglycol FCC at $25^{\circ} \mathrm{C}$. Filled circles are experimental data from [Unpublished]. Empty circles are the validation experiments of this work. The white areas are the formulation windows according to Poutons' LFCS (see Table I).

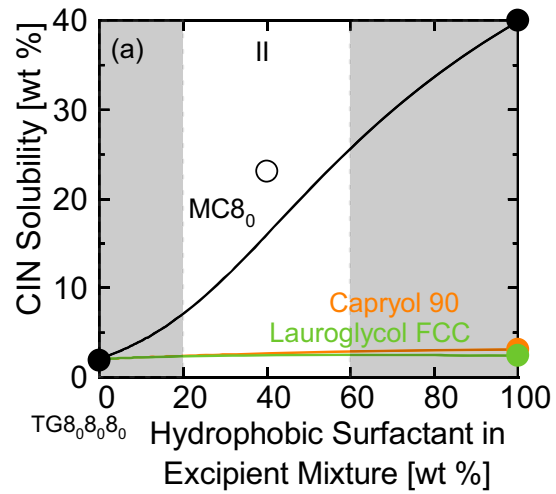

Capryol 90 to an excipient mixture of TPGS1000 and carbitol could further enhance the IND solubility.

Figure 6 shows the predicted IND solubilities in the ternary excipient mixture comprising Capryol 90 (hydrophobic surfactant), TPGS1000 (hydrophilic surfactant) and carbitol (cosolvent) together with the concentration boundaries of type IV formulations as well as the area of TPGS1000 crystallization.

The boundaries of the type IV-formulation window were again found to intersect with the TPGS1000-crystallization region. Thus, the TPGS1000 concentration in the excipient mixtures should not exceed $60 \mathrm{wt} \%$ to avoid crystallization. The IND solubility was predicted to increase with increasing amounts of carbitol to up to $9.3 \mathrm{wt} \%$ in an excipient mixture of $50 \mathrm{wt} \%$ TPGS 1000 and $50 \mathrm{wt} \%$ carbitol. It can thus be concluded that an addition of Capryol 90 decreases the IND solubility in formulations with TPGS 1000 and carbitol. However, it was predicted that the solubility decrease upon addition of Capryol 90 is less pronounced than the decrease upon addition of $\mathrm{TG}_{0} \mathrm{8}_{0} 8_{0}$ (compare Fig. 5).

Figure 7 compares the solubilities of FFB and IND in all considered formulation types (types I to IV). It clearly shows that the screening results for FFB and IND are very different for the different formulations types of the LFCS. The solubility of IND is predicted to be very low in type I and II formulations and is distinctively enhanced in type III and IV formulations. In contrast, the predicted FFB solubility is already high in $\mathrm{TG}_{0} 8_{0} 8_{0}$ and only slightly increases in the type II formulation. For types III and IV formulations, the FFB solubility is predicted to decrease due to the lower $\mathrm{TG}_{0} 8_{0} 8_{0}$ concentrations in the excipient mixtures.

In a next step, the screening approach was extended to investigate whether the solubility of IND and NAP could further be enhanced by choosing another cosolvent than carbitol. Figure 8 compares IND and NAP solubilities in excipient mixtures of $50 \mathrm{wt} \%$ TPGS 1000 and $50 \mathrm{wt} \%$ of either carbitol, ethanol, or PEG400.

The screening results reveal the highest IND and NAP solubilities in an excipient mixture of TPGS1000 and carbitol. For both APIs, the solubility is predicted to be lowest in the excipient mixture of TPGS1000 and PEG400. E.g., the NAP solubility is predicted to decrease from an TPGS1000/ carbitol mixture to a TPGS1000/PEG400 mixture by approximately $30 \%$. Thus, carbitol is recommended here as cosolvent in type IIIb or IV formulations for IND and NAP.

These results underline the importance of investigating the influence of different cosolvents. Especially for type IIIb and IV formulations which contain up to $50 \mathrm{wt} \%$ cosolvents in the excipient mixture, identifying the most-suitable cosolvent is very important.

\section{Formulating the Brick-Dust APIs GRI and GLI}

GRI and GLI are denoted as brick-dust APIs [3] as their solubility in any excipient mixture is extremely low and it is
Fig. 3 API solubility of (a) FFB and (b) IBU in mixtures of $\mathrm{TG} 8_{0} 8_{0} 8_{0}$ and $\mathrm{MC}_{0}$, Capryol 90, or Lauroglycol FCC at $25^{\circ} \mathrm{C}$. Filled circles are experimental values measured with HPLC [Unpublished].Empty circles are the validation experiments of this work. The white areas mark Poutons' LFCS formulation window (see Table I).
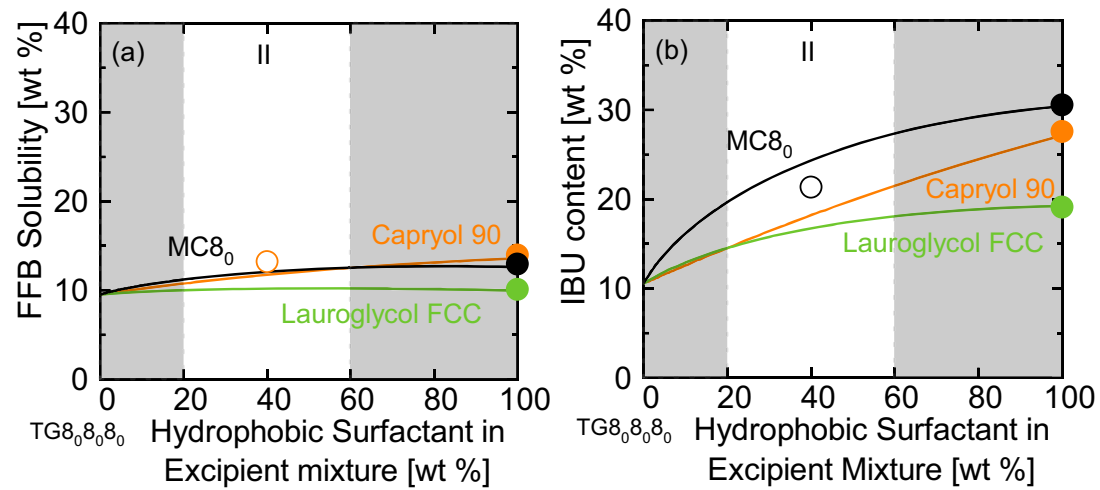
Fig. $4 P Z Q$ solubility in mixtures of (a) $\mathrm{TG} 8_{0} 8_{0} 8_{0} / \mathrm{MC} 8_{0}, \mathrm{TG} 8_{0} 8_{0} 8_{0} /$ Capryol 90 and $\mathrm{TG} 8_{0} 8_{0} 8_{0} /$ Lauroglycol FCC and (b) Capmul MCM/MC8, Capmul MCM/ Capryol 90 and Capmul MCM/ Lauroglycol FCC at $25^{\circ} \mathrm{C}$. Symbols are experimental literature values measured with HPLC

[Unpublished]. The white areas mark Poutons' LFCS formulation window (see Table I)

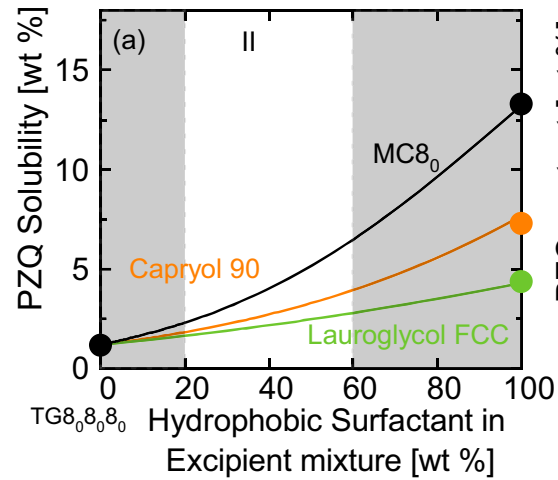

most challenging to find appropriate formulations for these API candidates. Figure 9 summarizes the screening results for GRI and GLI formulations from all LFCS types and the solubility gain, which can be achieved by each of these.

As it can be seen in Fig. 9, the solubility of GRI can distinctively be enhanced by a type IIIb formulation (PC-SAFT: $1.6 \mathrm{wt} \%$ ) compared to the GRI solubility in type I formulations. The experimental solubility $(1.1 \mathrm{wt} \%)$ was found in very good agreement with this screening result. Although this value is almost $900 \%$ higher than the GRI solubility in pure TG8 $8_{0} 8_{0} 8_{0}(0.1 \mathrm{wt} \%)$, the GRI solubility is still quite low. No further gain of the GRI solubility was predicted when formulating it in a type IV formulation (PC-SAFT predicted solubility in $15 \mathrm{wt} \%$ Capryol 90, $35 \mathrm{wt} \%$ TPGS1000 and $50 \mathrm{wt} \%$ carbitol: $1.2 \mathrm{wt} \%$ ). In a recent work, also other excipient mixtures were examined to find higher GRI solubilities [36]. However, among all systems considered in that work, the maximum GRI solubility was only found to be $1.5 \mathrm{wt} \%$ which compares to the values found above for the type IIIb formulation.

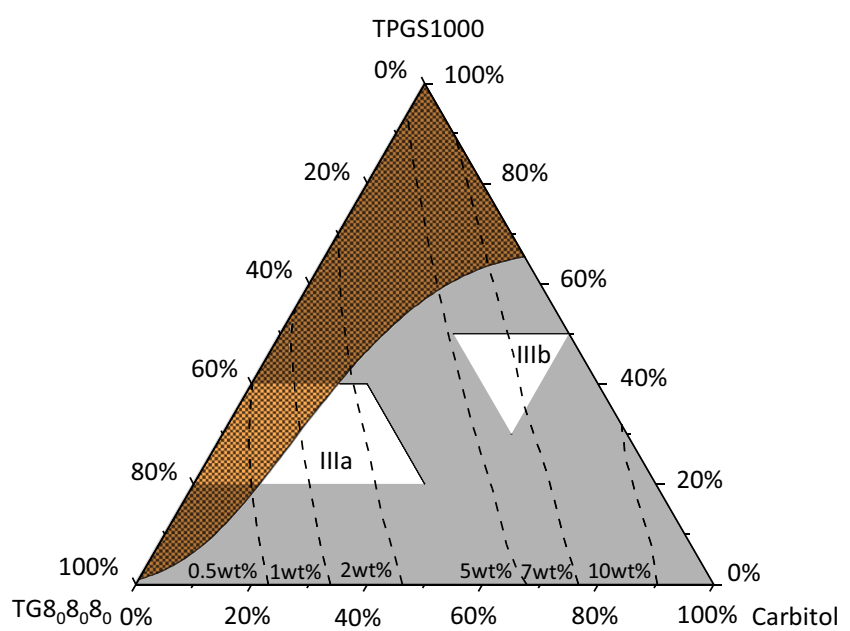

Fig. 5 IND solubility in TG8, $8_{0} 8_{0} / \mathrm{TPGSI000/carbitol} \mathrm{mixtures} \mathrm{at} 25{ }^{\circ} \mathrm{C}$. The squared area is the TPGSI 000 crystallization area. Dashed lines are PCSAFT predictions of constant IND solubility. White areas mark the formulation windows for LFCS type IIla and IIIb formulations (see Table I).
In case of GLI (Fig. 9b), the predicted solubility for all formulation types is even lower than the one of GRI. As before, the GLI solubility is predicted to increase for more hydrophilic formulation types (IIIb and IV). The experimental solubility in the IIIb formulation was found to be $0.6 \mathrm{wt} \%$, which agrees very well with the predicted ones. Although the GLI solubility in the type IIIb formulation shows a gain of more than $8000 \%$ compared to the type I formulation (see Fig. 1), the GLI solubility does not exceed about $1 \mathrm{wt} \%$. However, the experimental GLI solubility of $0.5 \mathrm{wt} \%$ might be sufficient for LBDDS applications as the dose in a commercial tablet is only 1 to $5 \mathrm{mg}$ [37]. This dose would be achieved in $1 \mathrm{~g}$ LBDDS of type IIIb or IV according to the screening performed in this work as well as its experimental validation.

Table VII lists the $\log \mathrm{P}$ values of the ten APIs considered in this work as well as the formulation types recommended from the screening in this work. Obviously, we do not observe a correlation between the $\log \mathrm{P}$ value of the APIs and the recommended formulation type as sometimes discussed in

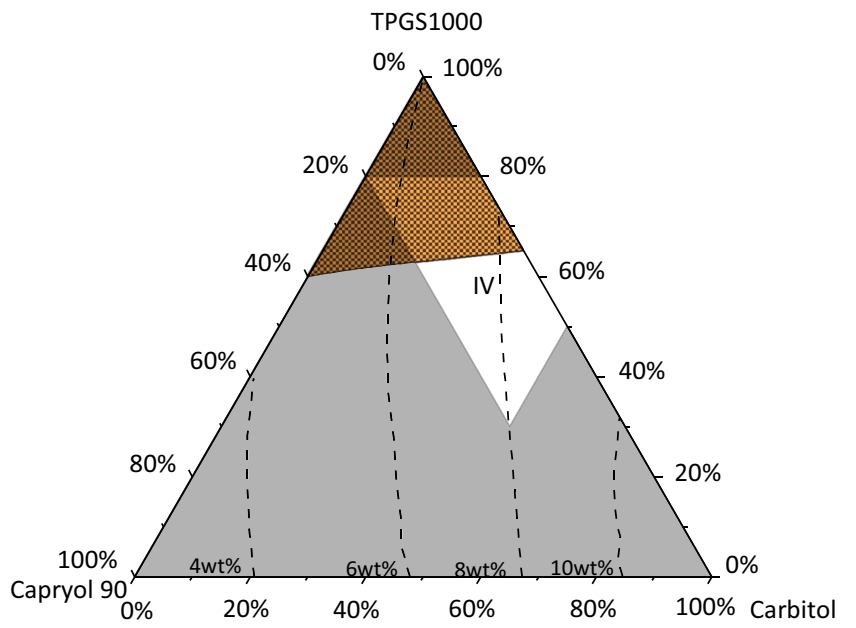

Fig. 6 IND solubility in TG8 $8_{0} 8_{0} 8_{0} / \mathrm{TPGSI000/carbitol} \mathrm{mixtures} \mathrm{at} 25{ }^{\circ} \mathrm{C}$. The squared area is the region where the TPGSI000 crystallizes. Dashed lines are PC-SAFT predictions of constant IND solubility. The white area marks the formulation window for LFCS type IV formulations (see Table I). 


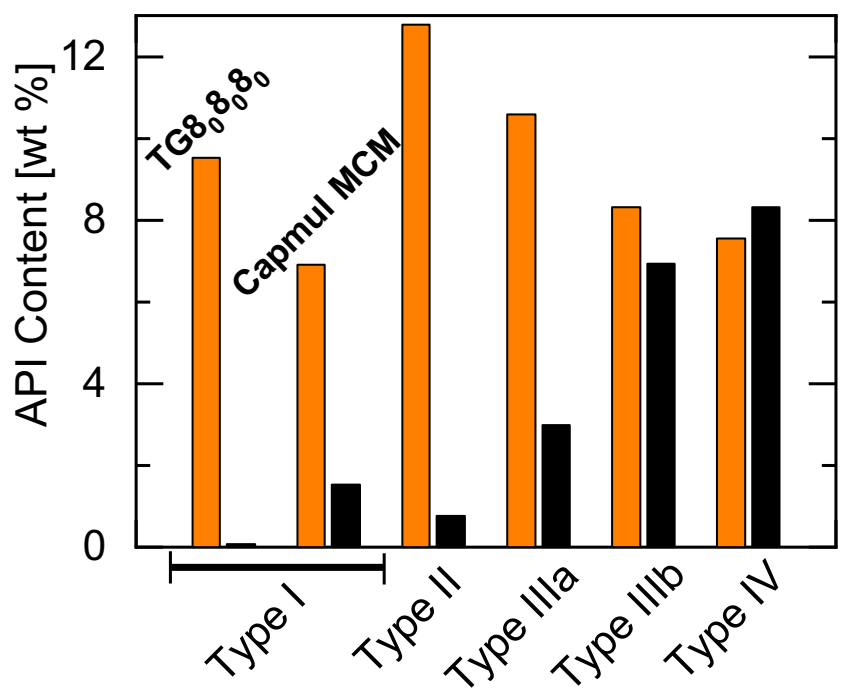

Fig. 7 PC-SAFT predicted solubility of FFB (left bars) and IND (right bars) in the different LFCS formulation types at $25^{\circ} \mathrm{C}$. Excipient mixtures contain: pure TG $8_{0} 8_{0} 8_{0}$ or Capmul MCM (type I); 40 wt $\%$ TG $8_{0} 8_{0} 8_{0}$ and 60 wt $\%$ Capryol 90 (type II), 40 wt\% TG8 8080 , 20 wt\% TPGSI000, and 40 wt\% carbitol (type IIla); I 5 wt\% TG8 $88_{0} 8_{0}, 35$ wt\% TPGSI000, and 50 wt\% carbitol (type IIIb), I 5 wt\% Capryol 90, 35 wt\% TPGSI000, and 50 wt\% carbitol (type IV).

literature [1]. It is thus not reasonable to choose formulation types for APIs based on their $\log \mathrm{P}$ value only. Instead, it is strongly recommended to screen the solubility of a given API on a case-to-case basis considering the specific interactions between the API and the components of an excipient mixture.

\section{CONCLUSIONS}

An in-silico solubility screening of ten model APIs was conducted to identify appropriate excipient mixtures for lipidbased drug delivery systems according to the lipid-based

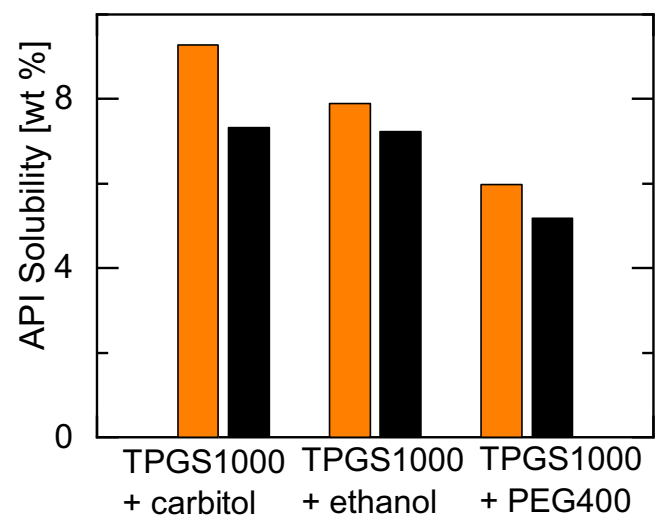

Fig. 8 Screening results for the solubility of IND (left bars) and NAP (right bars) in excipient mixtures of $50 \mathrm{wt} \%$ TPGSI000 and $50 \mathrm{wt} \%$ cosolvent (carbitol, ethanol, PEG400) at $25{ }^{\circ} \mathrm{C}$. API solubilities were predicted with PC-SAFT. classification system developed by Pouton. Using this screening approach, promising formulation types for eight out of the ten model APIs were found, namely

- Type I: fenofibrate, ibuprofen, praziquantel and carbamazepine

- Type II: cinnarizine and felodipine

- Types IIIb and IV: naproxen and indomethacin.

The solubilities of griseofulvin and glibenclamide were also predicted to be best in type IIIb and type IV formulations. However, they remained very low $\left(<1.6 \mathrm{wt}^{\mathrm{o}} \%\right)$ even in these formulations.

No correlation was found between the $\log \mathrm{P}$ value of the considered APIs and the recommended formulation type. Obviously just considering $\log \mathrm{P}$ is not sufficient. Instead, specific molecular interactions between an API and the components of the excipient mixture need to be considered on a case-by-case basis in the development of lipid-based drug delivery systems. Thus, in-silico screening based on thermodynamic modeling is a powerful tool to rapidly determine promising lipid-based drug delivery systems. It can be extended to additional excipients or APIs and thus allows to drastically decrease the number of experiments required for subsequent in vitro or in vivo tests.

\section{ACKNOWLEDGMENTS AND DISCLOSURES}

This work has been supported by Deutsche Forschungsgemeinschaft (DFG) with Grant SA700/20 (Gottfried Wilhelm Leibniz Prize awarded to Gabriele Sadowski).

Table VII APIs considered in this work, their logP values and recommended formulation types. Formulation type 0 denotes brick dust APIs

\begin{tabular}{llll}
\hline API & logP & formulation type & reference \\
\hline GRI & 2.2 & 0 & {$[14]$} \\
PZQ & 2.7 & I & {$[14]$} \\
CBZ & 2.7 & I & {$[14]$} \\
NAP & 2.8 & Illb or IV & {$[14]$} \\
FEL & 3.6 & || & {$[14]$} \\
IBU & 4.0 & I or II & {$[38]$} \\
GLI & 4.1 & 0 & {$[14]$} \\
IND & 4.2 & Illb or IV & {$[14]$} \\
FFB & 5.1 & I & {$[14]$} \\
CIN & 5.5 & II & {$[14]$} \\
\hline
\end{tabular}



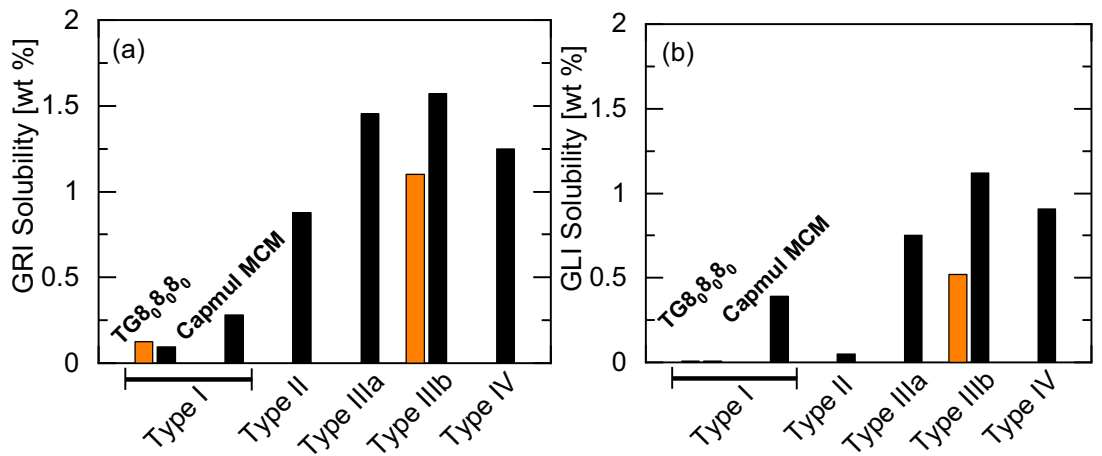

Fig. 9 Solubility screening results of (a) GRI and (b) GLI at $25^{\circ} \mathrm{C}$ comparing the solubility in excipient mixtures of each formulation type. Right bars are the PCSAFT predictions, left bars are validation experiments. The excipient mixtures contain either pure TG8 $8_{0} 8_{0} 8_{0}$ (type I); $60 \mathrm{wt} \% \mathrm{TG} 8_{0} 8_{0} 8_{0}$, and $40 \mathrm{wt} \% \mathrm{MC} 8_{0}$ (type II); 40 wt\% TG8,8,8, 20 wt\% TPGSI 000, and 40 wt\% carbitol (type IIla); I 5 wt\% TG8,8,8, 35 wt\% TPGSI 000, and 50 wt\% carbitol (type IIIb), I 5 wt\% Capryol 90, 35 wt\% TPGSI000, and 50 wt\% carbitol (type IV).

\section{FUNDING}

Open Access funding enabled and organized by Projekt DEAL.

Open Access This article is licensed under a Creative Commons Attribution 4.0 International License, which permits use, sharing, adaptation, distribution and reproduction in any medium or format, as long as you give appropriate credit to the original author(s) and the source, provide a link to the Creative Commons licence, and indicate if changes were made. The images or other third party material in this article are included in the article's Creative Commons licence, unless indicated otherwise in a credit line to the material. If material is not included in the article's Creative Commons licence and your intended use is not permitted by statutory regulation or exceeds the permitted use, you will need to obtain permission directly from the copyright holder. To view a copy of this licence, visit http://creativecommons.org/licenses/by/4.0/.

\section{REFERENCES}

1. Ditzinger F, Price DJ, Ilie A-R, Köhl NJ, Jankovic S, Tsakiridou G, et al. Lipophilicity and hydrophobicity considerations in bioenabling oral formulations approaches - a PEARRL review. J Pharm Pharmacol. 2019;71(4):464-82.

2. Kalepu S, Nekkanti V. Insoluble drug delivery strategies: review of recent advances and business prospects. Acta Pharm Sin B. 2015;5(5):442-53.

3. Müllertz A, Ogbonna A, Ren S, Rades T. New perspectives on lipid and surfactant based drug delivery systems for oral delivery of poorly soluble drugs. J Pharm Pharmacol. 2010;62(11):1622-36.

4. Porter GJH, Pouton GW, Guine JF, Charman WN. Enhancing intestinal drug solubilisation using lipid-based delivery systems. Adv Drug Deliv Rev. 2008;60(6):673-91.

5. Kuentz M. Lipid-based formulations for oral delivery of lipophilic drugs. Drug Discov Today Technol. 2012;9(2):97-104.

6. Pouton CW. Key issues when formulating hydrophobic drugs with lipids. Bull Tech Gattefosse. 1999;92:41-50.
7. Pouton CW. Lipid formulations for oral administration of drugs: non-emulsifying, self-emulsifying and 'self-microemulsifying' drug delivery systems. Eur J Pharm Biopharm. 2000;11:93-8.

8. Holm R. Bridging the gaps between academic research and industrial product developments of lipid-based formulations. Adv Drug Deliv Rev. 2019;142:118-27.

9. Brinkmann J, Huxoll F, Luebbert C, Sadowski G. Solubility of pharmaceutical ingredients in triglycerides. Eur J Pharm Biopharm. 2019;145:113-20.

10. Gautschi N, Bergström CAS, Kuentz M. Rapid determination of drug solubilization versus supersaturation in natural and digested lipids. Int J Pharm. 2016;513(1-2):164-74.

11. Alsenz J, Kuentz M. From quantum chemistry to prediction of drug solubility in glycerides. Mol Pharm. 2019;16(11):4661-9.

12. Persson LC, Porter CJH, Charman WN, Bergström CAS. Computational prediction of drug solubility in lipid based formulation excipients. Pharm Res. 2013;30(12):3225-37.

13. Brinkmann J, Rest F, Luebbert C, Sadowski G. Solubility of pharmaceutical ingredients in natural edible oils. Mol Pharm. 2020;17(7):2499-507.

14. Alskär LC, Porter CJH, Bergström CAS. Tools for early prediction of drug loading in lipid-based formulations. Mol Pharm. 2016;13(1):251-61.

15. Niederquell A, Dujovny G, Probst SE, Kuentz M. A relative permittivity approach for fast drug solubility screening of solvents and excipients in lipid-based delivery. J Pharm Sci. 2019;108(10):345760.

16. Hong J, Hua D, Wang X, Wang H, Li J. Solid-liquid-gas equilibrium of the ternaries ibuprofen + Myristic acid + CO 2 and ibuprofen + Tripalmitin + CO 2. J Chem Eng Data. 2010;55(1): 297-302.

17. Lehmkemper K, Kyeremateng SO, Degenhardt M, Sadowski G. Influence of low-molecular-weight excipients on the phase behavior of PVPVA64 amorphous solid dispersions. Pharm Res. 2018;35(1): 25.

18. Williams HD, Trevaskis NL, Charman SA, Shanker RM, Charman WN, Pouton CW, et al. Strategies to address low drug solubility in discovery and development. Pharmacol Rev. 2013;65(1):315-499.

19. Shrestha H, Bala R, Arora S. Lipid-based drug delivery systems. J Pharm. 2014;2014:1-10.

20. Gross J, Sadowski G. Perturbed-chain SAFT: An equation of state based on a perturbation theory for chain molecules. Ind Eng Chem Res. 2001;40(4):1244-60.

21. Prudic A, Ji Y, Sadowski G. Thermodynamic phase behavior of API/polymer solid dispersions. Mol Pharm. 2014;11(7):2294-304. 
22. Brinkmann J, Luebbert C, Zaitsau DH, Verevkin SP, Sadowski G. Thermodynamic modeling of triglycerides using PG-SAFT. J Chem Eng Data. 2019;64(4):1446-53.

23. Prausnitz JM, Lichtenthaler RN, de Azevedo EG. Molecular thermodynamics of fluid-phase Equilibria. 2nd ed. Englewood Cliffs: Prentice-Hall; 1986.

24. Watterson S, Hudson S, Svärd M, Rasmuson AC. Thermodynamics of fenofibrate and solubility in pure organic solvents. Fluid Phase Equilib. 2014;367:143-50.

25. Luebbert C, Huxoll F, Sadowski G. Amorphous-amorphous phase separation in API/polymer formulations. Molecules. 2017;22(2): 296.

26. Lange L, Sadowski G. Polymorphs, hydrates, Cocrystals, and Cocrystal hydrates: thermodynamic modeling of theophylline systems. Cryst Growth Des. 2016;16(8):4439-49.

27. Paus R, Ji Y, Vahle L, Sadowski G. Predicting the solubility advantage of amorphous pharmaceuticals: a novel thermodynamic approach. Mol Pharm. 2015;12(8):2823-33.

28. Paus R, Ji Y, Braak F, Sadowski G. Dissolution of crystalline pharmaceuticals: experimental investigation and thermodynamic modeling. Ind Eng Chem Res. 2015;54(2):731-42.

29. Paus R, Hart E, Ji Y, Sadowski G. Solubility and caloric properties of Cinnarizine. J Chem Eng Data. 2015;60(8):2256-61.

30. Gross J, Sadowski G. Application of the perturbed-chain SAFT equation of state to associating systems. Ind Eng Chem Res. 2002;41(22):5510-5.

31. Ruether F, Sadowski G. Modeling the solubility of pharmaceuticals in pure solvents and solvent mixtures for drug process design. J Pharm Sci. 2009;98(11):4205-15.
32. Stoychev I, Galy J, Fournel B, Lacroix-Desmazes P, Kleiner M, Sadowski G. Modeling the phase behavior of PEO-PPO-PEO surfactants in carbon dioxide using the PG-SAFT equation of state: application to dry decontamination of solid substrates. J Chem Eng Data. 2009;54(5):1551-9.

33. Prudic A. Phase behavior of polymer based pharmaceutical formulations [PhD thesis]. Dortmund: TU Dortmund University; 2015.

34. Lange L, Sadowski G. Thermodynamic modeling for efficient Cocrystal formation. Cryst Growth Des. 2015;15(9):4406-16.

35. Paus R. Solubility and Dissolution of Pharmaceuticals [ $\mathrm{PhD}$ thesis]. Dortmund: TU Dortmund University; 2015.

36. Arida AI, Al-Tabakha MM, Hamoury HAJ. Improving the high variable bioavailability of Griseofulvin by SEDDS. Chem Pharm Bull. 2007;55(12):1713-9.

37. Jurasovic M, Bouvier V. Gelbe Liste online. Neu-Isenburg: Medizinische Medien Informations GmbH; 2020.

38. Avdeef A, Box KJ, Comer JE, Hibbert C, Tam KY. pH-metric $\log \mathrm{P} 10$. Determination of liposomal membrane-water partition coefficients of ionizable drugs. Pharm Res. 1998;15(2):209-15.

Publisher's Note Springer Nature remains neutral with regard to jurisdictional claims in published maps and institutional affliations. 